\title{
Peirce y lo incognoscible. Respuesta a Damiani
}

\section{Daniel Kalpokas*}

Resumen: El presente artículo responde algunas objeciones que Damiani, en su trabajo "Comunidad, realidad y pragmatismo", efectúa a un artículo anterior mío sobre lo incognoscible en Peirce: "Lo incognoscible y los límites del sentido". Señalo que nuestros desacuerdos conciernen principalmente a dos puntos: (1) si tiene sentido sostener que no podemos saber si hay incognoscibles, y (2) si cabe defender la tesis de Peirce ("Lo absolutamente incognoscible es absolutamente inconcebible") sin comprometerse con el idealismo. Al argumentar por una respuesta afirmativa al primer punto y por una negativa al segundo, intento justificar una actitud agnóstica respecto de lo incognoscible: la de que no podemos saber si hay incognoscibles.

Palabras clave: Peirce, Damiani, incognoscible, realismo, idealismo

Abstract: This article replies to Damiani's objections to a previous paper of mine about the incognizable in Peirce: "The Incognizable and the Bounds of Sense". I point out that our disagreements are mainly about two points: (1) whether it has any sense to assert that we cannot know if there are incognizables, and (2) whether Peirce can defend his thesis ("The absolutely incognizable is absolutely inconceivable") without presupposing the idealism as a thesis about the nature of reality. I argue in favor of a positive answer for the first point, and a negative one for the second, and thus I try to justify an agnostic attitude with respect to the incognizable: we cannot know whether there are incognizables.

Key Words: Peirce, Damiani, incognizable, realism, idealism

* Doctor en Filosofía por la Universidad de Buenos Aires, Investigador Adjunto del CONICET, Profesor Adjunto Regular en la Facultad de Filosofía y Humanidades de Universidad Nacional de Córdoba, miembro plenario de la Sociedad Argentina de Análisis Filosófico (SADAF) e integrante del Grupo de Acción Filosófica (Gaf) de la Universidad de Buenos Aires. Email: dkalpokas@gmail.com. 
En la última parte de su artículo "Comunidad, realidad y pragmatismo", Damiani presenta varias objeciones a un artículo mío en el cual discutía algunas afirmaciones y argumentos de Peirce sobre lo incognoscible ${ }^{2}$. En este trabajo quiero responder a tales objeciones.

En mi artículo "Lo incognoscible y los límites del sentido" examinaba críticamente la tesis de Peirce según la cual "Lo absolutamente incognoscible es absolutamente inconcebible" (CP 5.310) ${ }^{3}$. Dicha tesis está dirigida contra todos aquellos que pretenden sostener que hay o puede haber aspectos incognoscibles de la realidad (el caso prototípico tal vez sea la noción kantiana de "cosa en sî"). Frente a Peirce, mi objetivo era defender una actitud agnóstica sobre lo incognoscible: la de que no podemos saber si hay incognoscibles. Para ello, examinaba varios textos peirceanos para tratar de mostrar que los argumentos contra la idea de lo incognoscible no son concluyentes. Entendía que los textos considerados daban lugar a, por lo menos, dos interpretaciones posibles: a veces Peirce parece sugerir que el término o concepto "incognoscible" carece de significado, y a veces parece sostener, en cambio, que aquello que se postula como incognoscible carece de sentido. Damiani considera que es obvio que Peirce está sosteniendo esta última tesis $\mathrm{y}$, por eso, piensa que pierdo el tiempo al tratar de refutar la primera posibilidad hermenéutica. No pretendo discutir aquí qué es obvio y qué no lo es, de modo que remito al lector a los pasajes de la obra de Peirce citados en mi artículo original para que juzgue por sí mismo si son tan claros como piensa Damiani ${ }^{4}$.

Considero que dos son los puntos de disidencia entre Damiani y yo: (1) si tiene sentido hablar de lo incognoscible como algo que la comunidad

1 Alberto Damiani, “Comunidad, realidad y pragmatismo", Tópicos, N ${ }^{\circ} 19-20$, jul/dic 2010.

2 Daniel Kalpokas, "Lo incognoscible y los límites del sentido", en Di Gregori, C. y Herbrard, A. (comp.), Peirce, Scbiller, Dewey y Rorty. Usos y revisiones del pragmatismo clásico, Buenos Aires, Ediciones del Signo, 2009.

${ }^{3}$ Las citas corresponden a Charles S. Peirce, Collected Papers, vol. 1-5, Cambridge, Harvard University Press, 1931-1935 (en el resto del artículo como CP).

${ }^{4}$ Cf. por ejemplo CP 5.255, 5.310, 5.257. 
indefinida de investigadores nunca podría conocer; y (2) el realismo entendido como una tesis ontológica. Comienzo con el primer punto y tomo la interpretación de los textos peirceanos que Damiani considera como la "obviamente" correcta.

(1)- El texto principal considerado (aunque no el único) es "Questions Concerning Certain Faculties Claimed For Man". Mi estrategia consistió en analizar críticamente los tres argumentos que Peirce aduce a favor de la tesis según la cual lo que es incognoscible "no puede tener significado" (CP 5.255). El primer argumento puede reconstruirse como sigue:

Premisa 1- Obtenemos todas nuestras concepciones por medio de cogniciones que se dan en la experiencia.

Premisa 2- La experiencia no puede manifestar lo que es absolutamente incognoscible ${ }^{5}$.

Premisa 3- El significado de un término es la concepción (de los efectos prácticos concebibles) que el término transmite.

Conclusión: Lo que es incognoscible no puede tener significado.

En primer lugar, si es cierto que la primera premisa, junto con la premisa 3, impone restricciones a lo que puede tener significado o puede concebirse, entonces parece que otros conceptos podrían correr la misma suerte que aquello que se considera como incognoscible. Por ejemplo, uno podría usar las mismas premisas para, mutatis mutandis, sostener que "lo que es infinito" no puede tener significado. Dudo que Peirce haya querido llevar

${ }^{5}$ Esta premisa corre el riesgo de trivialidad. En efecto, por "experiencia" Peirce parece estar entendiendo "experiencia cognoscitiva", con lo cual está diciendo que la experiencia - una forma de conocimiento- no puede manifestar lo que no puede conocerse. Parece que queda abierta siempre la posibilidad de que lo incognoscible se manifieste en la experiencia aun cuando no podamos detectarlo en cuanto tal. Estas consideraciones no estaban en mi artículo original y por ello no volveré a ellas. 
su argumento tan lejos. En segundo lugar, si bien puede concederse que en cierto sentido la conclusión es verdadera (no se puede atribuir ningún predicado empírico a lo que se postula como incognoscible), también parece cierto que en otro sentido no lo es. Siguiendo a Chalmers, en mi artículo original distingo dos sentidos de "concebir". Concebir algo positivamente sólo requiere que podamos concebir una situación particular en la que S (una afirmación cualquiera) sea el caso (por ejemplo, "Hay incognoscibles"). Por otro lado, concebir negativanente algo requiere, en cambio, que $S$ no pueda ser rechazada a priori o que no sea contradictoria ${ }^{6}$ (por ejemplo, en el caso de que "Hay incognoscibles" no sea contradictoria en sí misma). En estos dos sentidos hablar de lo que es incognoscible parece tener significado. En su trabajo, Damiani no dice nada al respecto. $5.257)^{7}$.

El segundo argumento de Peirce puede reconstruirse así (CP.

Premisa 1- "Incognoscible” es una cognición.

Premisa 2- "Incognoscible" expresa (es un concepto de) la no cognoscibilidad.

Premisa 3- Hay una contradicción entre 1 y 2.

Conclusión: "Incognoscible" es de la forma "A y no A".

En mi artículo original argumentaba que la premisa 3 parece ser simplemente falsa, pues entre las premisas 1 y 2 no hay ni una contradicción formal (como parece decir Peirce) ni una contradicción semántica (si las hubiera), pues es manifiesto que si atendemos al contenido del concepto "incognoscible", no hallaremos contradicción alguna.

${ }^{6} \mathrm{La}$ distinción entre concebibilidad positiva y negativa se encuentra en Chalmers, David, "Does Conceivability Entail Possibility?", en Szabó Gendler T. y Hawthorne, J (eds), Conceivability and Possibility, Oxford, Clarendon Press, 2002.

${ }^{7}$ Este es uno de los pasajes en los que me parece claro que Peirce está hablando del concepto "incognoscible", y no de lo que es incognoscible. 
Tampoco puede decirse que exista una contradicción entre el hecho de que "incognoscible" sea una cognición y el hecho de que exprese la incognoscibilidad. ¿Qué tipo de contradicción podría haber aquí entonces? Caritativamente sugerí que teníamos que pensar, no en el concepto "incognoscible", sino en afirmaciones que pretenden referirse a una realidad incognoscible, y seguidamente sugerí que tal vez podía entenderse la contradicción involucrada aquí como una contradicción pragmática. Este es el único mérito que Damiani me reconoce, sólo que para criticar, seguidamente, la manera en que presento la cuestión. Veamos con cierto detenimiento este punto.

Sostuve que quien afirma (1) "Hay incognoscibles" con pretensión de saber, comete una contradicción pragmática, pues al afirmar que hay incognoscibles está pretendiendo conocer algo que reconoce que no puede conocerse. Como dije en mi artículo, "No se puede sostener consistentemente la pretensión de conocer algo que se dice que no se puede conocer" 8 . Uno tiene la pretensión de saber que p cuando, por lo menos, cree que $\mathrm{p}$, pretende que $\mathrm{p}$ es verdadera, $\mathrm{y}$ pretende tener justificación para creer que $\mathrm{p}^{9}$. Ahora bien, imprudentemente pensé que podía explicitarse dicha pretensión mediante (2) "Sé que hay incognoscibles", lo cual motivó la atendible crítica de Damiani, lo que él denomina "el grave error" de confundir el verbo "saber" con un verbo performativo ${ }^{10}$. Efectivamente, en

${ }^{8}$ Kalpokas, Daniel, "Lo incognoscible y los límites del sentido", p. 129.

${ }^{9}$ El "por lo menos" señala un recaudo contra los contraejemplos Gettier. Si tales contraejemplos son correctos, señalan la insuficiencia de las conocidas condiciones que definen el saber proposicional; no cuestionan, pues, su carácter necesario. Por tanto, si hubiera que agregar alguna condición más a la noción de conocimiento para sortear los contraejemplos Gettier, eso no afectaría a lo que estoy sosteniendo. ${ }^{10} \mathrm{Ha}$ habido desarrollos sobre verbos performativos que distinguen performativos estrictos y performativos en sentido extendido, y se ha sugerido que "Yo sé" es performativo en este último sentido (Cf. Chisholm, R., "J. L. Austin Philosophical Papers", Mind, 73, 289, p. 1-26). Cuando alguien dice "Yo sé", según Chisholm, no sólo está describiendo su estado psicológico en términos de conocimiento, sino que además está dando garantías de lo que sabe. En mi respuesta, no obstante, asumiré 
un sentido estricto puede decirse que el verbo "saber" no es performativo, pues por el mero hecho de decir "Yo sé que p" no sé que p. Con todo, la objeción de Damiani no invalida mi argumentación. En efecto, (3) "No hay incognoscibles" dicho con pretensiones de conocimiento, o (4) "Sé que no hay incognoscibles", y (5) "No es posible que haya incognoscibles" dicho con pretensiones de conocimiento, o (6) "Sé que no es posible que haya incognoscibles" son todas afirmaciones auto-contradictorias ${ }^{11}$. Uno no puede saber que no hay incognoscibles (ciertos rasgos, entidades, eventos, etc. de la realidad) si en verdad son incognoscibles, pues si son incognoscibles uno no puede saber nada acerca de ellos ${ }^{12}$. Damiani, que pretende defender a Peirce, no considera ninguna de estas afirmaciones, aunque debería hacerlo, pues si en verdad tales afirmaciones son autocontradictorias, y si en verdad son equivalentes a, o están implicadas por, la tesis de Peirce, entonces la tesis Peirce-Damiani sobre lo incognoscible es necesariamente falsa. Sin embargo, la afirmación que defiendo no es contradictoria en ningún sentido: (7) "No es posible saber si hay incognoscibles", dicho con pretensiones de conocimiento, o (8) "Sé que no es posible saber si hay incognoscibles". Por tanto, si bien la observación de Damiani es correcta, ello no toca en modo alguno mi tesis principal. Es más, no afecta mi crítica a la tesis Peirce-Damiani según la cual dicha tesis no hay ni puede haber incognoscibles- sería auto-contradictoria.

(2) El problema de lo incognoscible está conectado con el problema del realismo. Damiani cree que Peirce tiene razón al desechar por "oscura" la definición de lo real "como aquello cuyas características son

que puede concederse el punto a Damiani y que la distinción de Chisholm puede desestimarse en el presente contexto.

${ }^{11}$ Mientras que (3) y (5) parecen ser pragmáticamente auto-contradictorias, (4) y (6) parecen involucrar una contradicción lógica.

${ }^{12} \mathrm{El}$ argumento que sostendría la afirmación "Sé que no hay incognoscibles" es que carece de sentido - de acuerdo a la máxima pragmática- la postulación misma de algo que es incognoscible. Como señalo más adelante, esto supone que es lícito pasar de lo que la comunidad indefinida de investigadores considera inconcebible a la no existencia (o imposibilidad de existencia) de algo. Este salto de lo inconcebible a la afirmación ontológica antedicha es, me parece, cuestionable. 
independientes de lo que cualquiera puede pensar que son" (CP 5. 405). Esta es, naturalmente, una afirmación ontológica acerca de la naturaleza de la realidad. Aquí curiosamente las intuiciones de Damiani y las mías se invierten, pues la citada definición peirceana de la realidad me parece más clara que la versión supuestamente mejorada. Según Damiani, la oscuridad de esta primera definición de realidad reside en que "puede conducirnos a pensar que la realidad es totalmente independiente del pensamiento humano en general, en el sentido de que algunas propiedades de lo real serían por principio incognoscibles para el pensamiento humano" 13 . Ahora bien, es preciso notar que efectivamente la definición de realidad en cuestión puede y -agregaría yo- debe conducirnos a pensar que la realidad es independiente del pensamiento humano en general si es que uno pretende dar una definición realista de realidad. Precisamente, el realismo qua tesis ontológica entiende que la realidad física existe independientemente de nuestros pensamientos (incluso del pensamiento en general) ${ }^{14}$. El realista cree que dicha realidad existía antes de que hubiese pensamiento humano en el mundo, por ejemplo, y que seguirá existiendo mucho tiempo después de que desaparezcan los seres humanos en el universo. Piensa eso porque cree que el pensamiento humano en general no es constitutivo de la realidad física. Sin embargo, de esta afirmación ontológica no se sigue en modo alguno que -como afirma Damiani"algunas propiedades de lo real serían por principio incognoscibles para el pensamiento humano". Tal vez haya algunas propiedades de lo real que sean incognoscibles para nosotros, tal vez no; pero por el solo hecho de afirmar la independencia ontológica de la realidad física todavía no se sigue ningún compromiso con la incognoscibilidad. Hace falta un argumento que pruebe que existe tal conexión entre independencia ontológica e incognoscibilidad, y Damiani no ofrece tal argumento. Todo lo contrario, sigue a Peirce al afirmar que lo real no puede ser independiente del

13 Damiani, Alberto, "Comunidad, realidad y pragmatismo", p. 121.

${ }^{14}$ Sobre esta concepción del realismo, cf. Searle, J., La construcción de la realidad social, Barcelona, Paidós, 1997; Devitt, M., Realism and Truth, Princeton, Princeton University Press, 1997; y Kalpokas, D., "Realismo, relatividad conceptual e independencia ontológica”, Analítica, No 1, año 1, Lima, 2007. 
pensamiento en general. Pero esta afirmación de corte idealista, que sostiene que el pensamiento en general constituye al menos en parte la realidad física, es justamente un supuesto de la argumentación peirceana.

El último argumento de Peirce que es objeto de mi crítica aparece en el siguiente pasaje: "Frente a cualquier cognición, hay una realidad desconocida pero cognoscible; pero contra toda cognición posible, sólo existe lo autocontradictorio. En resumen, la cognoscibilidad (en su sentido más amplio) y el ser no son sólo metafísicamente lo mismo, sino que son términos sinónimos" (CP. 5.257).

$\mathrm{Si}$ bien esto aparece en "Questions concerning certain faculties claimed for man", creo que es legítimo decir que la tesis según la cual el ser y lo cognoscible son lo mismo también puede atribuirse a los textos posteriores de Peirce. El argumento que subyace a la afirmación de Peirce parece ser éste:

Premisa 1: Si algo es incognoscible, entonces es inconcebible.

Premisa 2: Si algo es inconcebible, entonces no puede existir.

Conclusión: Si algo es incognoscible, entonces no puede existir.

Este argumento es -a mi juicio- problemático por varias razones. En primer lugar, en la premisa 1 Peirce parece inferir, de la imposibilidad epistémica de conocer algo, la imposibilidad lógico-conceptual (la inconcebibilidad). Pero hay que probar que esto es así15. Como señalé oportunamente arriba, es posible concebir positiva y negativamente lo incognoscible. No resulta nada claro, pues, que la primera premisa sea verdadera.

Pero aun cuando lo fuera, hay que probar la verdad de la premisa 2 . $\mathrm{Y}$ el salto desde lo inconcebible a la no existencia parece cuestionable.

\footnotetext{
${ }^{15}$ La máxima pragmática, que establece una relación entre lo concebible y lo que podría revelarse en la experiencia (los efectos prácticos del objeto de nuestra concepción), sólo afirma tal conexión.
} 
Dado que la experiencia hace posible el conocimiento, algo inconcebible (si es incognoscible) jamás podría manifestarse en la experiencia ${ }^{16}$. Pero el que exista o no algo así no puede depender de nuestro conocimiento posible (al menos no si no asumimos de entrada el idealismo). Sólo asumiendo el idealismo (en este caso, la tesis según la cual la existencia de la realidad depende de la posibilidad de que sea conocida) puede concluirse que lo incognoscible no puede existir (o, como dice Peirce, que "la cognoscibilidad (...) y el $\operatorname{ser}(. .$.$) son términos sinónimos"). Sin embargo, la tesis idealista en$ cuestión es, como la tesis realista, una tesis metafísica (en el mismo sentido en que creo que Damiani habla de "realismo metafísico" cuando me lo atribuye). A menos que uno acepte el idealismo, no puede decirse que sea un rasgo necesario de la realidad el que sea cognoscible. Pero entonces, no podemos saber a priori que el ser y lo cognoscible tienen la misma extensión. No podemos saber que no hay incognoscibles.

Ahora bien, Damiani no discute este argumento, ni las consideraciones que hago en torno a cada premisa. Simplemente me atribuye -incomprensiblemente, desde mi punto de vista- "una dirección semanticista" que conduciría a la tesis de que el "ámbito de la realidad es más amplio que el del conocimiento posible"17. En realidad, no es eso lo que sostengo en mi trabajo, sino que, dado que el argumento de Peirce no muestra una equivalencia a priori entre ser y cognoscibilidad, "Es una posibilidad lógica que el reino del ser se extienda más allá de lo cognoscible"18. Damiani me atribuye la comisión de "falacia abstractiva", como si por el mero hecho de sostener el carácter independiente de la realidad uno necesariamente tuviera que dejar a un lado la dimensión pragmática del lenguaje. Tal vez tal acusación podría tener cierto asidero si yo estuviera comprometido con la tesis de que la realidad es incognoscible. Pero claramente no es ese el caso.

${ }^{16}$ En rigor de verdad, tal vez podría manifestarse en la experiencia sin que lo sepamos; pero, por mor de la simplicidad, concederé aquí el punto a Peirce.

${ }^{17}$ Damiani, Alberto, "Comunidad, realidad y pragmatismo", p. 138.

${ }^{18}$ Kalpokas, Daniel, "Lo incognoscible y los límites del sentido", p. 132. 
Ciertamente, sostengo que, sin contradecirse, uno puede hacer afirmaciones como "Hay incognoscibles" o "No hay incognoscibles" sin tener razones para justificar una u otra y, en definitiva, sin poder siquiera saber si alguna de esas afirmaciones es verdadera. También sostengo que, pese a ello, uno puede tener una idea acerca de cuáles son las condiciones que tales afirmaciones deberían satisfacer para ser verdaderas (que haya incognoscibles o no, respectivamente), pero, ¿por qué esto equivaldría a "pensar la verdad de las proposiciones con absoluta independencia de los actos de habla asertóricos"? ¿Acaso no estoy sosteniendo que podemos concebir las condiciones de verdad de tales afirmaciones? ¿Por qué, por el sólo hecho de que uno no pueda conocer la verdad de una afirmación, se sigue que está ignorando "la parte performativa de los actos de habla"? Damiani parece pensar que, si uno afirma que no se puede conocer el valor de verdad de una proposición, automáticamente separa a la proposición de las acciones lingüísticas. No veo cuál podría ser el argumento. Si digo "Hay incognoscibles", aunque no podamos saber si esa afirmación es verdadera, ¿no es obvio que he realizado un acto de habla afirmativo, cuyo contenido proposicional puede ser verdadero, y con conocidas condiciones de verdad que tal afirmación debería satisfacer para ser verdadera? ${ }^{19}$ La pregunta de

19 Siguiendo a Apel, Damiani sostiene que los actos de habla asertóricos, en su parte performativa, no sólo contienen implícitamente una pretensión de verdad, sino también "el presupuesto necesario de la posible resolución de la misma mediante el discurso argumentativo de la comunidad ideal e ilimitada", pp. 137 y 138. Así, la pretensión de verdad de cualquier afirmación estaría vinculada necesariamente al posible conocimiento de su verdad efectiva. Ahora bien, puesto que dicho conocimiento corre por cuenta de la comunidad ideal de hablantes y no por parte de los hablantes que aquí y ahora hacen las afirmaciones, estos últimos han de poder entender el significado de sus afirmaciones sin recurrir al conocimiento de la verdad de las mismas, es decir, antes de que la comunidad ideal pueda decir si tales afirmaciones son verdaderas o falsas. Dejando a un lado el problema de cómo uno podría saber a priori que necesariamente es posible conocer el valor de verdad de toda afirmación posible (algo que parece requerir un supuesto idealista de trasfondo), no me parece nada claro que dicho conocimiento desempeñe un papel esencial en la comprensión del significado de las afirmaciones. Más bien, parece mucho más simple pensar que, al menos para el caso de los actos 
Damiani es: ¿para quién sería verdadera tal afirmación? Si "Hay incognoscibles" remite, como sujeto epistémico, a la comunidad indefinida de investigadores humanos, la respuesta es: para cualquier sujeto de conocimiento que, si acaso existe, pueda conocer lo que nosotros no.

Finalmente, Damiani dice que me alejo de la concepción pragmática de la realidad "en la dirección de un realismo metafísico, que postula la posibilidad de una realidad por principio inaccesible para nosotros" 20 . Si por "metafísico" Damiani quiere decir "realismo ontológico", un tipo de realismo que sostiene que la realidad es independiente de nuestros pensamientos, entonces acepto el calificativo de "metafísico" para mi realismo, sólo que no veo nada malo en ello. Pues no creo que, como él dice, ello implique una desconexión de las nociones de verdad, conocimiento y realidad de las acciones lingüísticas. Como acabo de sugerir, ello no introduce la idea de "un ojo de Dios" que compara las proposiciones (cuya verdad nos sería incognoscible) con la realidad (incognoscible para nosotros), pues justamente estoy afirmando que no podemos saber si hay algún sujeto de conocimiento distinto de nosotros que pueda llevar a cabo esa tarea. Todo lo contrario, es la idea peirceana de opinión final la que introduce -aunque sea como ideal regulativo- la noción de "ojo de Dios". Es justamente el recurso a esa idea lo que le permite sostener a Peirce (y a Damiani) la tesis de que la realidad es, en principio, cognoscible.

En síntesis, las objeciones de Damiani no han sido dirigidas contra los principales argumentos que he esgrimido contra la tesis y argumentos de Peirce. Mi actitud agnóstica, por tanto, se mantiene. Decir "Sé que no hay (y no es posible que haya) incognoscibles" es auto-contradictorio. Y el argumento que supuestamente apoya tal afirmación -lo incognoscible es inconcebible a la luz de la máxima pragmática- supone un salto injustificado desde lo inconcebible (para la comunidad indefinida de investigadores) a lo que no hay ni puede haber en el mundo. En cambio, decir "Sé que no es

de habla asertóricos, la comprensión de las condiciones de verdad juega el papel requerido para la comprensión de los actos de habla mencionados.

${ }^{20}$ Damiani, Alberto, “Comunidad, realidad y pragmatismo", pp. 138-139. 
posible saber si hay incognoscibles" no es auto-contradictorio. Esa es justamente la afirmación que defiendo. Buena parte de las observaciones hechas aquí sobre el significado de las expresiones lingüísticas que involucran el término "incognoscible", junto con sus implicaciones realistas, no son más que corolarios de la posición que defiendo sobre lo incognoscible. 